\title{
A self-calibration scheme for Extended Frequency-Band-Decomposition sigma-delta ADC
}

\author{
Philippe Benabes, Ali Beydoun
}

Received: date / Accepted: date

\begin{abstract}
Frequency-Band-Decomposition (FBD) is a good candidate to be used to increase the bandwidths of ADC converters based on sigma-delta modulators for software and cognitive radio applications where we need to convert wide bandwidths. Each modulator processes a part of the band of the input signal which is then passed through a digital filter. In the case of large mismatches in the analog modulators, a new solution, called Extended Frequency-Band-Decomposition (EFBD) can be used. As an example, this solution can allow for a $4 \%$ error in the central frequencies without significant degradation of its performance when the digital processing part is paired with the analog modulators. A calibration of the digital part is thus required to reach these theoretical performances. This paper will focus on a self-calibration algorithm for an EFBD. The algorithm helps minimize the quantization noise of the EFBD and helps to flatten the signal transfer function.
\end{abstract}

Keywords sigma-delta - bandpass · analog-to-digital conversion · filter bank · frequency-band-decomposition · self-calibration

\section{Introduction}

The Frequency-Band-Decomposition (FBD) [1], [2], [3] is a natural way to widen the bandwidth of sigma-delta converters [4], using parallel bandpass modulators, where each modulator processes a part of the band of the input signal [5]. The main issue of this process is its sensitivity to the central frequencies of the bandpass modulators. Extended Frequency-Band-Decomposition (EFBD) [5] makes it possible

P. Benabes

SUPELEC, SSE Department, F91192 GIF/YVETTE

Tel.: +33-1-69851419

Fax: +33-1-69851429

E-mail: philippe.benabes@supelec.fr

A.Beydoun

TELECOM-ParisTech, COMELEC Department,

46 rue Barrault, F75634, Paris

Tel.: +33-6-15971018

E-mail: beydoual@yahoo.fr 
to convert a wideband signal, using two extra continuous-time bandpass modulators. This solution is able to adapt to the analog mismatches of the modulators, in order to minimize the quantization noise and to allow for the correct reconstruction of the input signal. These analog mismatches are caused by variations in the manufacturing processes. The transfer function should be as close as possible to a simple delay (minimum in-band ripple and linear phase).

As in many conversion systems, a calibration of digital part of the EFBD becomes unavoidable. Many sigma-delta topologies need a digital calibration. For example, the mismatch effects in MASH topologies could be reduced by using an on-line or an off-line calibration [6] or a background coefficient estimation [7]. The non-linearities induced in the DAC of multi-bit modulators can also be reduced by the use of calibration [8], [9]. Time-Interleaved solutions need calibration as the gain and offset errors induce spurious-tones [10].

A parallel lowpass sigma-delta solution also needs a calibration. As in the case of Time-Interleaved converters, gain and offset errors induce unwanted tones that can be minimized by adequate calibration [11], [12]. Such a calibration is performed by applying constant signals to each modulator and deducing the gain and offset from the filter outputs.

One of the advantages of the FBD solution is that linear mismatches do not produce spurious tones. They only result in an in-band ripple and in an increase of the quantization noise. Using continuous-time modulators helps increase the sampling frequency, and induces natural filtering in the working band of each modulator. On the other hand, local STF corrections must be performed together with phase adjustments in the case of an EFBD. The calibration proposed in [12] for FBD can only deal with offset and gain, which is not sufficient for EFBD solutions. An algorithm that can calibrate all the parameters of the digital part of an EFBD without requiring heavy computing resources is proposed in this paper.

The second section of this paper gives the main elements of the optimization of the quantization noise of an EFBD: first, the theoretical elements and second, the calibration method. The output mean power is minimized by modifying the bandwidths of the filters, the input simply being grounded. The third section deals with the signal transfer function (STF) flattening. Finally, the fourth section concludes with the complexity and the calibration time of the whole algorithm.

\section{Optimization of EFBD Noise Transfer Function}

\subsection{Theory}

An EFBD is composed of $N+2$ parallel sigma-delta modulators (Fig. 1 - part A), where $N$ is the number of modulators required to process the input signal band $\left[f_{1}, f_{2}\right]$. Two extra modulators are used in the case of large analog mismatches so that the useful band $\left[f_{1}, f_{2}\right]$ remains within the working band of the FBD. The output of all channels are merged by a digital system (part B) to reconstruct the input signal.

The major benefit of the EFBD architecture is that it allows to increase the conversion bandwidth with a linear increase of the power consumption (the total power consumption is $N+2$ times the power consumption of one modulator) whereas 


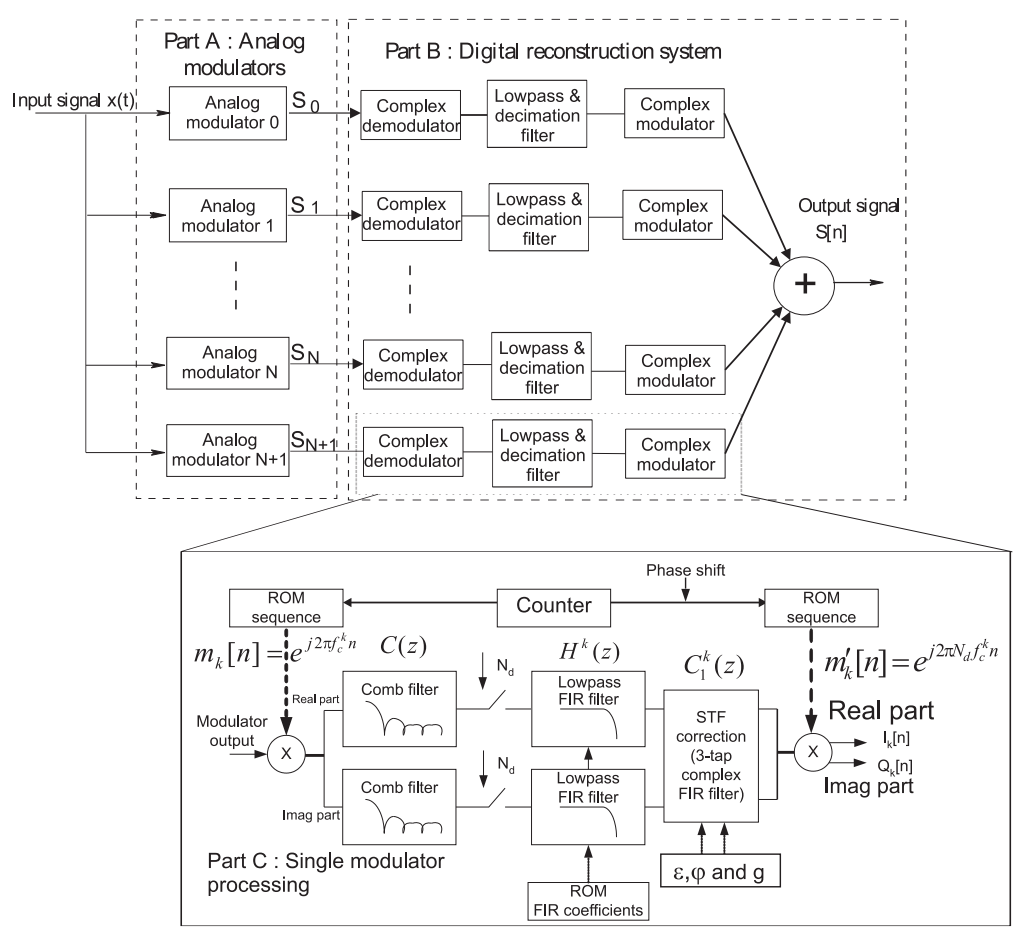

Fig. 1 Extended Frequency Band Decomposition architecture

with a single bandpass sigma-delta modulator, the power consumption increases exponentially while increasing the bandwidth [13].

Continuous-time modulators (Figure 1, part A) are used to overcome difficulties related to the frequency limitations of the discrete-time integrators that are built with switched capacitor circuits [14]. They can be designed from their discrete-time counterparts by using the method published in [14]. The sigma-delta modulator used in this paper is a third order continuous time bandpass modulator with a 3-bit quantizer using the classic cascaded topology as shown in Fig 2 (the purpose of this paper is not the topology of the analog modulator ; some more adequate topologies can be found in [16] or [15]).

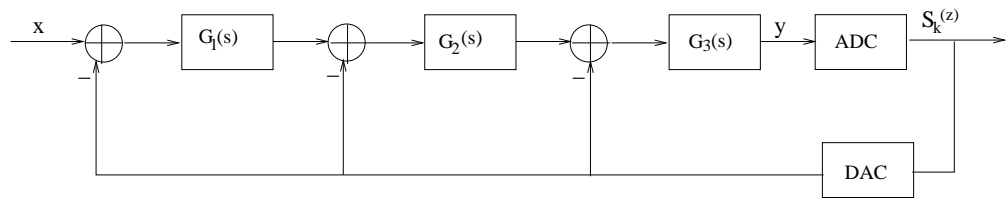

Fig. 2 Third order continuous-time bandpass sigma-delta modulator. 
The expected performance of each modulator can be evaluated from the in-band noise power given by [4]:

$$
P_{\text {Noise }}=\frac{\pi^{2 L} e^{2}}{(2 L+1)(O S R)^{2 L+1}}
$$

where :

$\mathrm{L}$ is the modulator order,

$e^{2}$ is the quantization noise power,

$O S R$ is the oversampling ratio.

The order of the modulator was set at three due to its chaotic behavior when no signal is applied at the input. This characteristic is very important to ensure a good running of the proposed calibration algorithms. We choose 3 as the number of bits of the ADC inside the loop to fulfill the expected performances of our application. Moreover, with 3-bit quantizer, the linear model of the quantizer [17] is often verified.

The digital processing associated with each modulator is composed of a complex demodulation process that brings the signal to baseband (demodulation frequencies are denoted $f_{C}^{k}$ ), of a comb filter that performs a decimation, of a FIR filter $H^{k}(z)$ that removes the out-of-band noise, of a signal transfer function correction filter $C_{1}^{k}(z)$ and of a modulation. The complete digital processing for one modulator is summarized in Fig. 1 - part C. The frequencies used for demodulation and modulation are expressed as rational numbers so that the sequences are finite and can be stored in a ROM [5].

The Noise Transfer Function (NTF) optimization of the system consists in using, for each frequency band, the modulator that has the best Signal-to-Noise Ratio (SNR). As the Signal Transfer Function (STF) is usually quite flat in the working band of the modulator, the modulator which is to be used for each frequency is the one whose noise power density is the lowest at this frequency.

Fig. 3 gives as an example, the noise power densities of each modulator $\left(N T F^{k}\right)$, where all resonator frequencies differ from their ideal values by a constant value plus random mismatches (modeling process non-idealities). The $\mathrm{Q}$ factors of the filters are equal to 50. The boundaries between the bands of each modulator are represented by vertical lines. In this example $\left(f_{1}=0.2, f_{2}=0.3\right)$, the $10^{\text {th }}$ modulator $\left(\Sigma \Delta_{9}\right)$ is not used as its band is completely outside the signal band.

The global system quantization noise power is the sum of the contributions of each modulator: $P_{N T F_{t}}=\sum_{k=0}^{N+1} P_{N T F^{k}}$.

$$
P_{N T F^{k}}=\int_{f=-\frac{1}{2}}^{\frac{1}{2}}\left|H^{k}\left(e^{2 j \pi N_{d}\left(f-f_{C}^{k}\right)}\right)\right|^{2}\left|N T F^{k}\left(e^{2 j \pi N_{d} f}\right)\right|^{2} \Gamma_{k}(f) d f
$$

$N_{d}$ is the decimation ratio, $\Gamma_{k}(f)$ is the quantization noise power spectral density and can be assumed constant [17]. $H^{k}(f)$ is the $k^{\text {th }}$ FIR filter transfer function. It is assumed that the comb decimation filter sufficiently removes the folded noise produced by aliasing. 


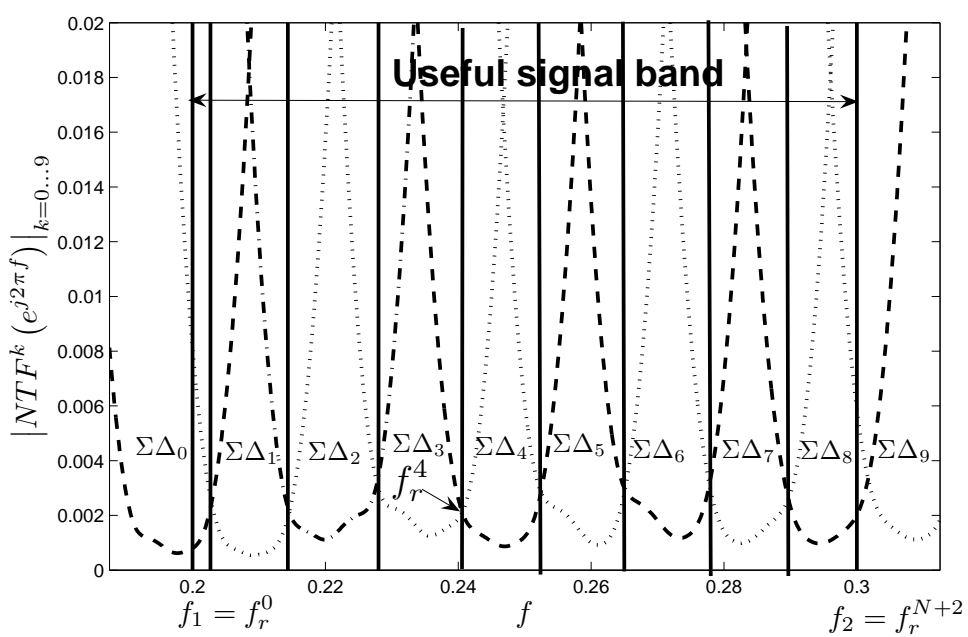

Fig. 3 Boundaries with non-ideal modulators

Fig. 4 gives an example of the magnitude of the product $\left|H^{k}\left(e^{2 j \pi N_{d}\left(f-f_{C}^{k}\right.}\right)\right|^{2}\left|N T F^{k}\left(e^{2 j \pi N_{d} f}\right)\right|^{2}$ for $k=4$. Most of the noise energy remaining after filtering lies between the modulator boundaries (vertical lines).

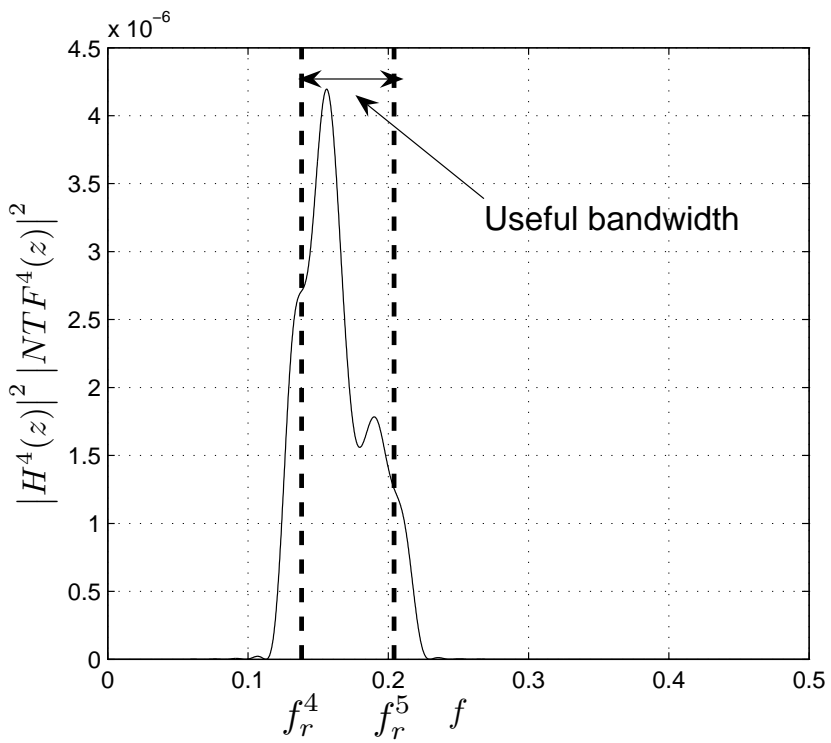

Fig. 4 Noise power spectral density after filtering

Thus it can be supposed that each FIR filter suppresses all the out-of-band noise, which leads to: 


$$
P_{N T F^{k}}=\int_{f=f_{r}^{k}}^{f_{r}^{k+1}}\left|N T F^{k}\left(e^{2 j \pi f}\right)\right|^{2} \Gamma(f) d f
$$

where $f_{r}^{k}$ and $f_{r}^{k+1}$ being the actual boundaries of the useful bands of the modulator $k$. All sub-bands are continuous with $f_{r}^{0}=f_{1}$ and $f_{r}^{N+2}=f_{2}$.

The quantization noise power spectral density is given by [17] :

$$
\Gamma_{k}(f)=\Gamma=\frac{1}{3 \times 4^{N b i t}},
$$

where Nbit is the number of bits of the ADC within each sigma-delta modulator. The global noise power can be expressed by:

$$
P_{N T F_{t}}=\Gamma \sum_{k=0}^{N+1} \int_{f=f_{r}^{k}}^{f_{r}^{k+1}}\left|N T F^{k}\left(e^{2 j \pi f}\right)\right|^{2} d f
$$

Let $f_{t}^{k}$ be the constant frequency chosen as the middle of the band of each modulator $k$. Each integral is split into two terms that have at least one constant boundary. The first and the last term in the sum are separated as they are constant.

$$
\begin{aligned}
& P_{N T F_{t}}=\Gamma \int_{f=f_{1}}^{f_{t}^{0}}\left|N T F^{0}\left(e^{2 j \pi f}\right)\right|^{2} d f \\
& +\Gamma \sum_{k=1}^{N+1} \underbrace{\left(\begin{array}{c}
\int_{f=f_{1}}^{f_{r}^{k}} \\
f=f_{t}^{k-1} \\
f_{t}^{k} \\
+\int_{f=f_{r}^{k}}^{k}\left|N T F^{k-1}\left(e^{2 j \pi f}\right)\right|^{2} d f
\end{array}\right)}_{T^{k}\left(f_{r}^{k}\right)} \\
& +\Gamma \int_{f=f_{t}^{N+1}}^{f_{2}}\left|N T F^{N+1}\left(e^{2 j \pi f}\right)\right|^{2} d f
\end{aligned}
$$

The total noise power is then the sum of two constant values, plus $N+1$ terms, each of them depending on one of the boundaries $f_{r}^{1}$ to $f_{r}^{N+1}$. The non-constant term $\sum_{k=1}^{N+1} T^{k}\left(f_{r}^{k}\right)$ is then the term to be minimized in order to minimize $P_{N T F_{t}}$. Each boundary can then be optimized independently from the others. Fig. 5 shows as an example $\left|N T F^{3}\right|,\left|N T F^{4}\right|$ and the term $T^{4}\left(f_{r}^{4}\right)$ in Eq. (6) which is the criterion to be minimized where $f_{r}^{4}$ is involved. This criterion appears reasonably convex and smooth.

It was verified that this property remains true when taking the FIR filter effect into account, resulting in a behavior similar to that of Fig. 5. 


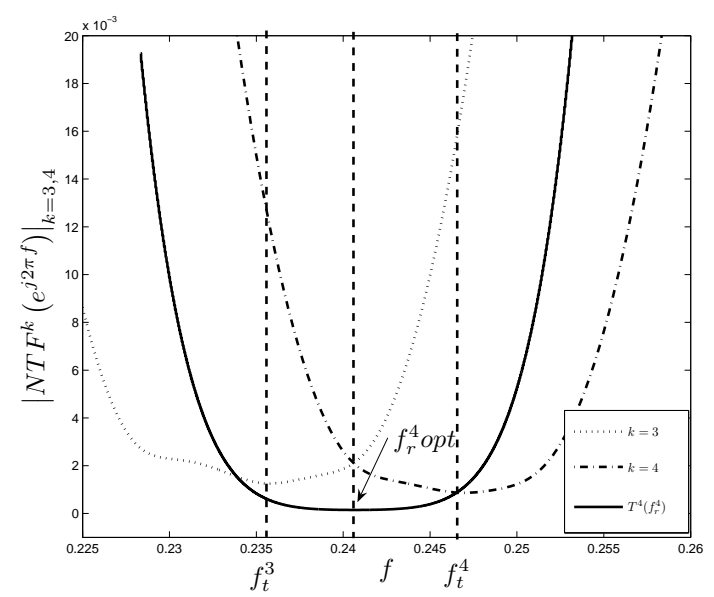

Fig. 5 Boundary optimization criteria

2.2 Noise transfer function calibration algorithm

The noise transfer function is optimized by tuning the boundaries between modulators $f_{r}^{k}, k=1 . . N+1$ from $f_{r}^{k-1}$ to $f_{r}^{k+1}$. The frequencies used for the modulator and demodulator are deduced from these values:

$$
f_{C}^{k}=\left(f_{r}^{k-1}+f_{r}^{k}\right) / 2
$$

and the bandwidths of the lowpass filters.

$$
\Delta f_{k}=\frac{f_{r}^{k}-f_{r}^{k-1}}{2} \text { for } k=1 . . N .
$$

It was shown in [5] that an error of $4 \%$ in the width of the sub-band $(0.05 \%$ of the sampling frequency) causes a resolution loss of less than 0.1 bit. Thus, the boundary frequency values $\left(f_{r}^{k}\right)$ can be quantized in this example with a step $q_{s}=F_{s} / 1024$. The whole band represents 102 steps and the bandwidth of each sub-band would be 12 or 13 steps if all modulators were ideal.

In order to get only the quantization noise at the output of the EFBD architecture, the input must be first grounded. The noise power produced by the quantization is then minimized by varying the values of the boundary frequencies $f_{r}^{k}$. The demodulation sequence and the lowpass FIR filters are fully determined by the knowledge of these values (7) and (8).

The adaptation scheme given in Fig. 6 was used. The scheme itself uses the processing of the digital part presented in Fig. 1. The estimate of the converter noise power is:

$$
\hat{P}=\frac{1}{N_{s}} \sum_{n=1}^{N_{s}} \sum_{k=1}^{N+2}\left(I_{k}[n]^{2}+Q_{k}[n]^{2}\right)
$$

$N_{s}$ is the number of samples used for the power estimation, $I$ and $Q$ are the outputs of each filter (Fig. 1) . 


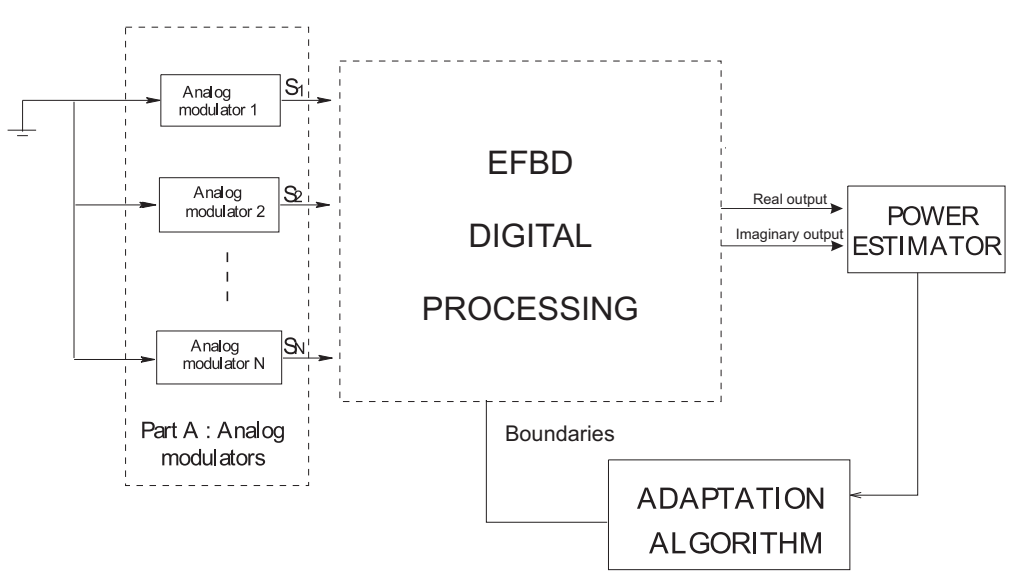

Fig. 6 Block diagram of the NTF optimization algorithm.

The proposed algorithm is based on a so-called relaxation method [18]. It does not require any operations except the calculation of the output power. Other faster algorithms could be used [19], but lead to more complex logic. Initial values are those obtained theoretically (i.e. typical design values). The algorithm changes iteratively the boundary value $f_{r}^{k}$ between $f_{r}^{k-1}$ and $f_{r}^{k+1}$ for $k=1$ to $N+1$ in each sequence. The first boundary remains $f_{1}$ and the last boundary remains $f_{2}$. Fig. 7 shows the flow diagram of this algorithm. The quantization noise power is estimated from a finite number of samples, leading to an error in the estimation. Due to this error, the algorithm will not converge to the optimum value in one step. It has to be applied several times to be sure to reach the optimum point. When $2^{12}$ samples are used for the quantization noise power calculation, two or three sequences are required to reach the optimum parameters.

This algorithm has been performed on a simulation of the modulator bank described in Fig. 3 with 10 modulators. The output signals of the modulators have been memorized so that the same outputs are used for each loop. The results are given in Fig. 8, which shows the resolution of the EFBD (calculated from the estimated noise power) after each iteration. It may be seen that the optimum value is reached at the end of the first sequence.

This algorithm has also been tested in a simulation where the output of the modulators were calculated for each iteration. The result is given in Fig. 9. It can be seen that the optimum is reached after three sequences. It has been verified by simulations that the convergence to the optimum value is also reached after three sequences for all kinds of mismatches (with systematic or random errors on the modulator's central frequencies). The method is therefore relevant.

It has been shown that with the Off-Line method the calibration was established with 9 iterations. This is due to the fact that the error produced by the noise power estimation is the same for each calculation step because the same data are considered. On the contrary, with the On-Line method, the estimation error varies at each calculation step. This leads to needing more iterations to converge to frequency values minimizing the noise power at the output as shown in figure 9. It was verified also that even if the linear model of the ADC inside the modulator loop [17] is not 


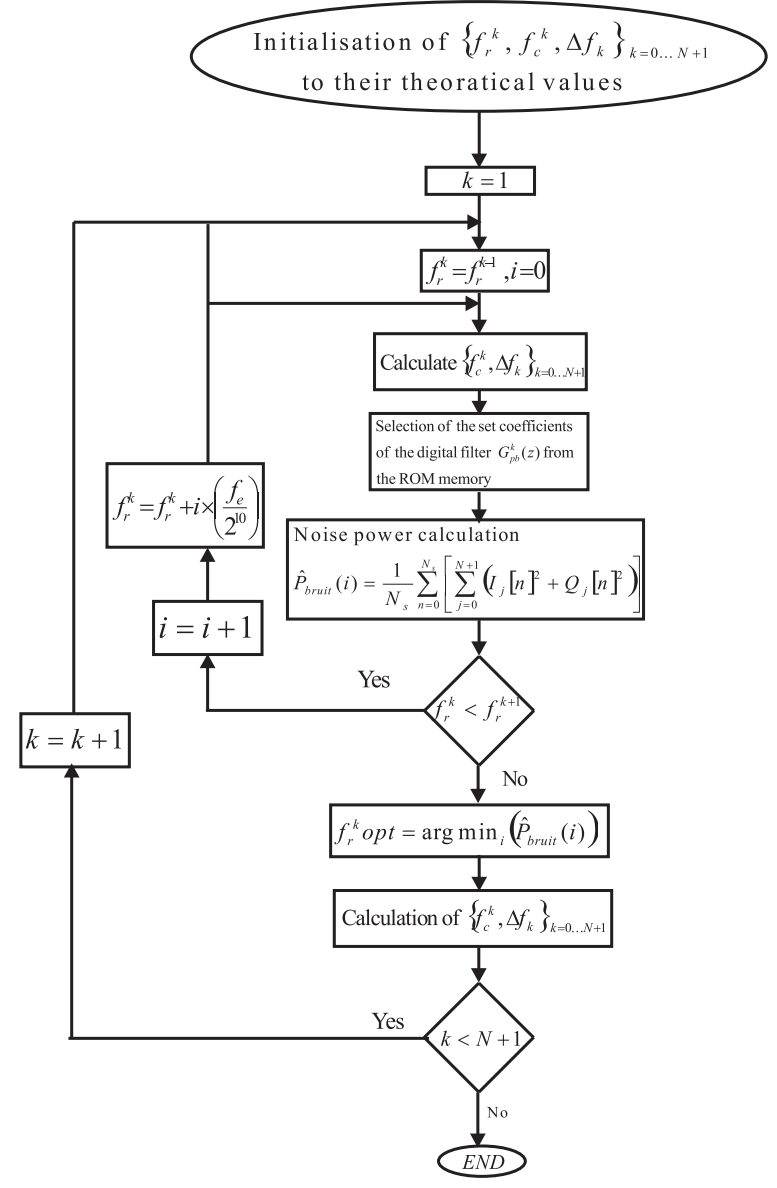

Fig. 7 Flow diagram of noise transfer function calibration algorithm.

verified, the proposed algorithm will converge to the optimal solution with the same run time.

With an $800 \mathrm{MHz}$ analog frequency, each noise power calculation performed from 4000 samples takes $5 \mu$ s to perform (the OSR of the system is equal to 5 ), and each sequence, in the worst case, takes approximately $1.12 \mathrm{~ms}$ (9 boundaries are varied among 25 values). The total NTF calibration time for 3 sequences can be estimated to $3.3 \mathrm{~ms}$.

\section{Optimization of the signal transfer function}

If the system was perfect, the output signal power would be constant when the input frequency varies. Unfortunately, the modulator transfer functions are not flat. They can be calculated by methods proposed in [20] and, usually, the modulus of the STF can be approximated by a parabola whose maximum is in the modulator band [5]. 


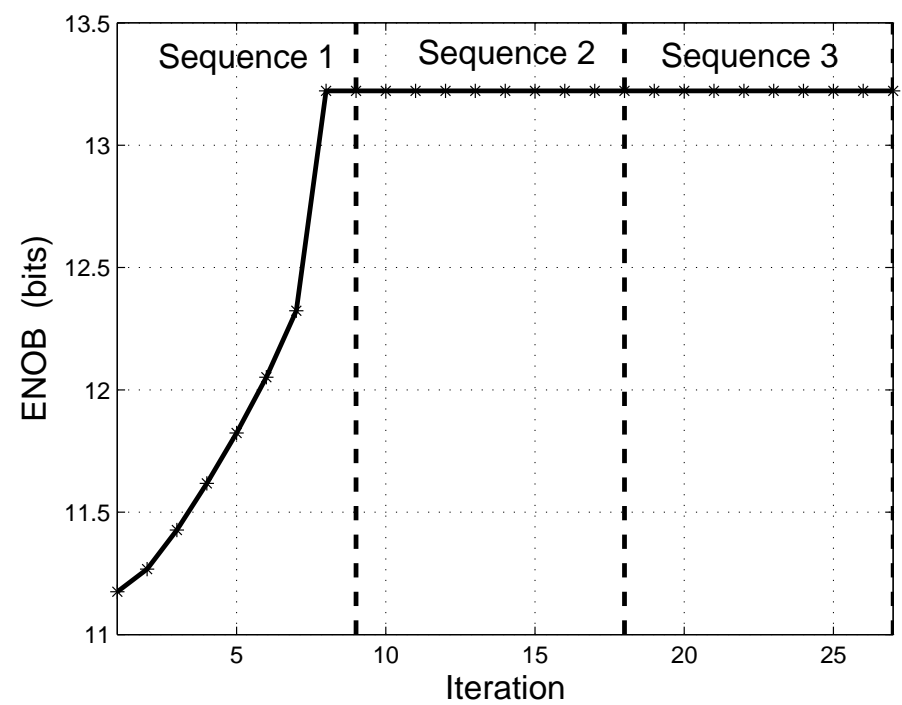

Fig. 8 Evolution of the Effective Number Of Bits (ENOB) during iterations -off line

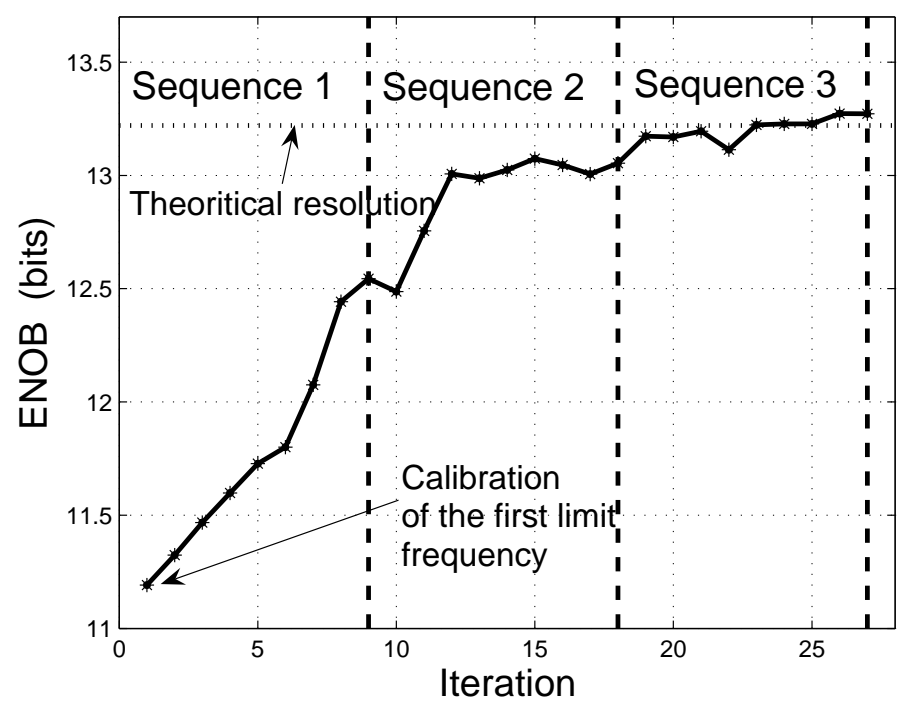

Fig. 9 Evolution of the Effective Number Of Bits (ENOB) during iterations -on-line

A corrective filter is applied here to each modulator to flatten its signal transfer function.

Furthermore, there is a phase shift between adjacent modulators. This phase shift is corrected by changing the phase of the modulation sequence used in the last stage of the digital processing [5]. 
The STF calibration is performed in two steps: first, to adjust the transfer function of each modulator, then adjust the phases between adjacent channels.

3.1 Flattening the STF of each modulator

Relying on a 2nd order polynomial approximation of the modulator in its working band, a $2 n d$ order complex filter was proposed in [5] to equalize the module of the $\mathrm{STF}$. This correction filter is expressed by:

$$
C_{1}^{k}(z)=g\left(-\varepsilon e^{-j 2 \pi \nu}+(1+2 \varepsilon) z^{-1}-\varepsilon e^{j 2 \pi \nu} z^{-2}\right)
$$

where $\varepsilon$ is the curvature of the parabola, $\nu$ is the difference between the center of the sub-band and the frequency for which the STF is maximum, and $g$ is the inverse of the maximum of the STF magnitude ${ }^{1}$.

In order to simplify the implementation of this filter, and assuming that the maximum of the modulator STF remains within its working band, the coefficient $\nu$ is small, and the filter can be approximated by :

$$
C_{1}^{k}(z) \approx g\left(-\varepsilon(1-j 2 \pi \nu)+(1+2 \varepsilon) z^{-1}-\varepsilon(1+j 2 \pi \nu) z^{-2}\right)
$$

let $\phi=2 \pi \nu$, the transfer function of the filter becomes :

$$
C_{1}^{k}(z) \approx g\left(-\varepsilon(1-j \phi)+(1+2 \varepsilon) z^{-1}-\varepsilon(1+j \phi) z^{-2}\right)
$$

Let $X(z)$ be the $z$ transform of the input complex signal of this filter $(X(z)=$ $\left.X_{R}(z)+j X_{I}(z)\right)$, the real and imaginary part of the output complex signal $Y(z)=$ $Y_{R}(z)+j Y_{I}(z)$ are expressed by :

$$
\begin{aligned}
Y_{R}(z)= & g(1+2 \varepsilon) X_{R}(z) z^{-1}-g \varepsilon X_{R}(z)\left(1-z^{-1}\right)^{2} \\
& +g \varepsilon \phi X_{I}(z)\left(1-z^{-2}\right) \\
Y_{I}(z)= & g(1+2 \varepsilon) X_{I}(z) z^{-1}-g \varepsilon X_{I}(z)\left(1-z^{-1}\right)^{2} \\
& -g \varepsilon \phi X_{R}(z)\left(1-z^{-2}\right)
\end{aligned}
$$

Assuming $\varepsilon^{\prime}=\varepsilon g$ and $\nu^{\prime}=g \varepsilon \phi$, the above equations can be written in the following way:

$$
\begin{aligned}
Y_{R}(z)= & \left(g+2 \varepsilon^{\prime}\right) X_{R}(z) z^{-1}-\varepsilon^{\prime} X_{R}(z)\left(1-z^{-2}\right) \\
& +\nu^{\prime} X_{I}(z)\left(1-z^{-2}\right) \\
Y_{I}(z)= & \left(g+2 \varepsilon^{\prime}\right) X_{I}(z) z^{-1}-\varepsilon^{\prime} X_{I}(z)\left(1-z^{-2}\right) \\
& -\nu^{\prime} X_{R}(z)\left(1-z^{-2}\right)
\end{aligned}
$$

The parameters $\left\{\varepsilon^{\prime}, \nu^{\prime}\right\}$ control the central frequency and the curvature of the frequency response of the correction filter $C_{1}^{k}(z)$. To illustrate this fact, Fig. 10 and Fig. 11 show the modulus of the STF after correction while varying $\nu^{\prime}$ and $\varepsilon^{\prime}$ independently.

The optimum values of parameters $\left\{\varepsilon^{\prime}, \nu^{\prime}, g\right\}$ depend on the useful bandwidth on each channel. As these bandwidths were recalculated by the algorithm presented in

\footnotetext{
1 this filter is applied to the demodulated signal
} 


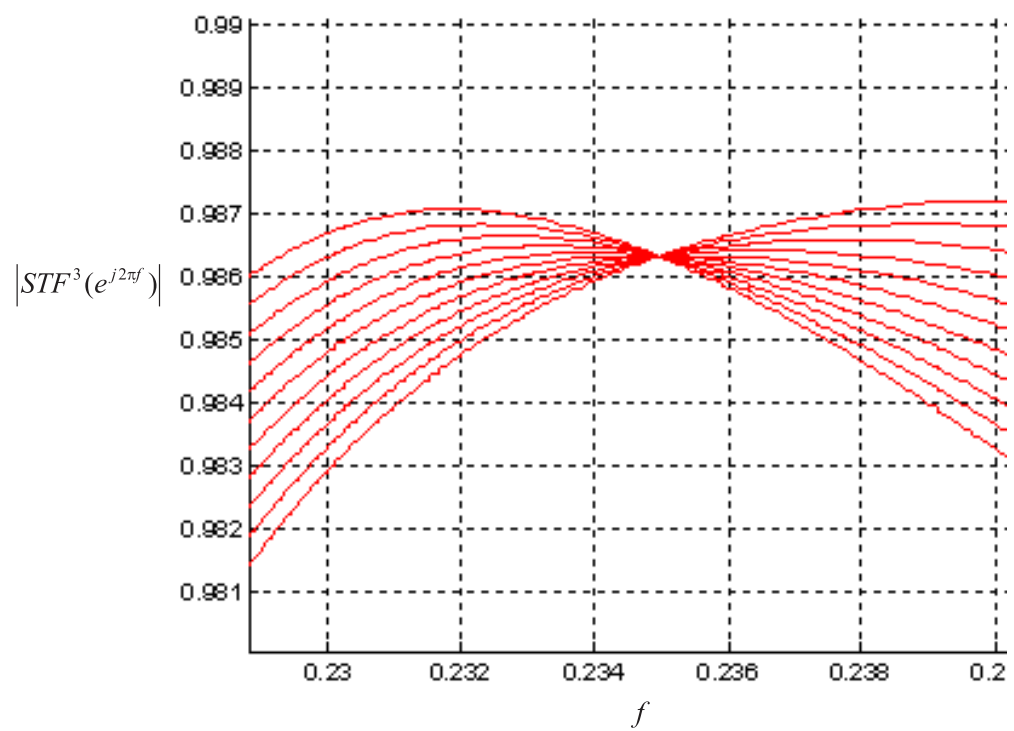

Fig. 10 Modulus of the STF after correction compared to the parameter $\nu^{\prime}$.

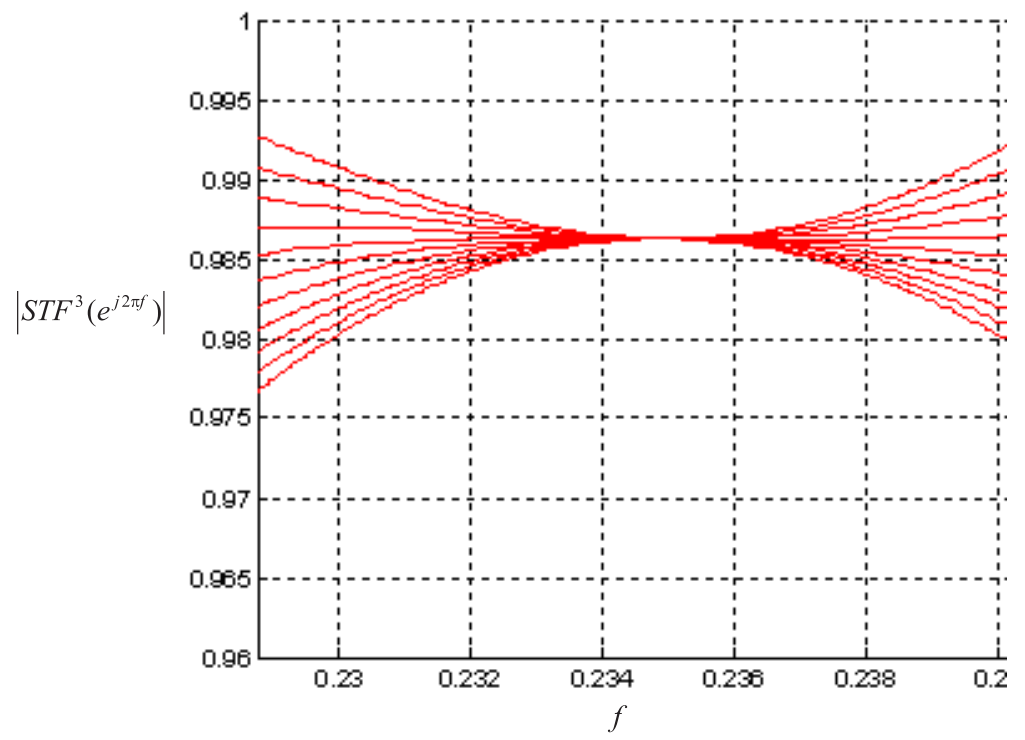

Fig. 11 Modulus of the STF after correction compared to the parameter $\varepsilon^{\prime}$

section 2.2 in order to minimize the global noise power at the output, it is therefore necessary to recalculate the values of $\left\{\varepsilon^{\prime}, \nu^{\prime}, g\right\}$ to ensure a good correction.

The criterion used by the search algorithm proposed in this article to find the optimum values $\left\{\varepsilon_{\text {opt }}^{\prime}, \nu_{\text {opt }}^{\prime}\right\}$ is to minimize the in-band ripples (the difference between the highest and the lowest amplitude in the working band) in each channel. 
The proposed algorithm is based upon a straightforward algorithm in which parameters $\varepsilon^{\prime}$ and $\nu^{\prime}$ vary independently in the respective intervals $\Delta \varepsilon^{\prime}$ and $\Delta \nu^{\prime}$ around their theoretical values. The dynamic range of each interval depends on the architecture of the modulator. Each interval is divided into $N_{v}$ values. The parameter $N_{v}$ controls the smoothing of the criterion and the required precision for the optimal values. Figure 12 shows the contour of the criterion to minimize with $\mathrm{N}_{\mathrm{v}}=16$. It can be noticed that the parameters $\varepsilon^{\prime}$ and $\nu^{\prime}$ are independent and a 16-split of each interval is sufficient to ensure a smoothing criterion. It was verified by simulations that the convergence of the algorithm is ensured for all cases in 2 or 3 iterations.

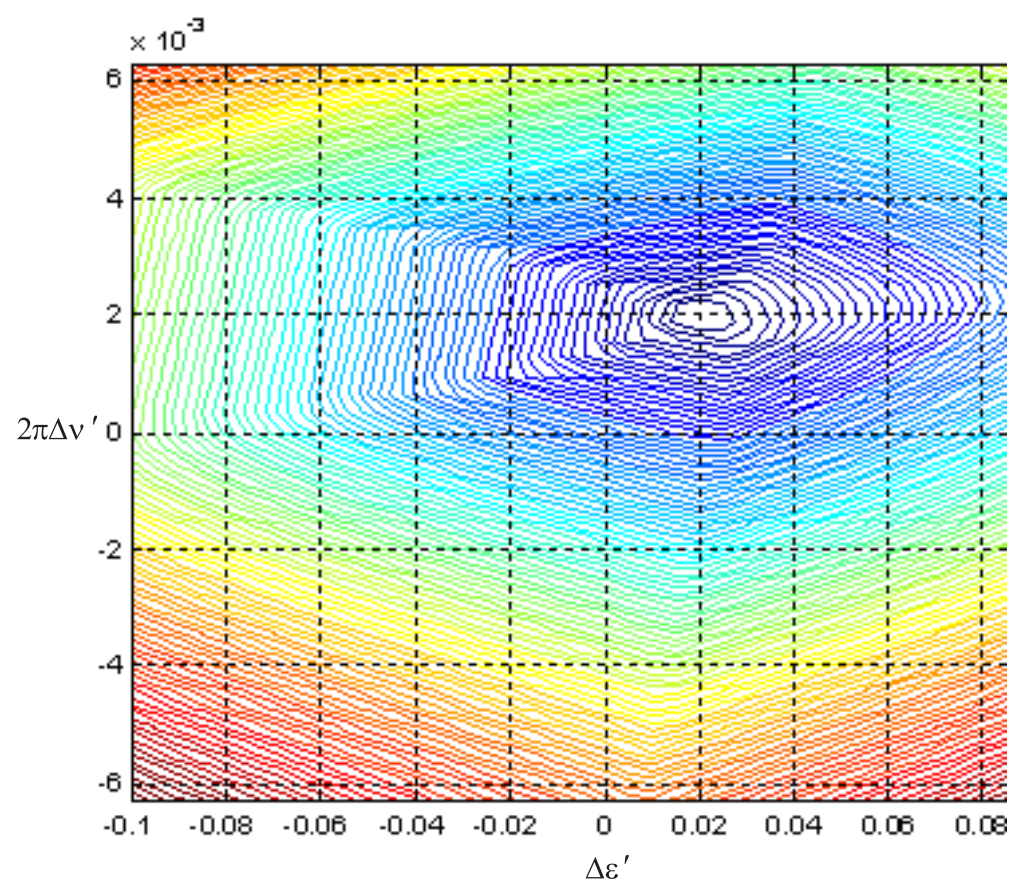

Fig. 12 Contour of the criterion to minimize

The implementation of this algorithm requires the measurement of the magnitude ripples in the working band at each iteration, so it is necessary to have a reference signal that has a constant spectral density in this band. A linear chirp signal (in which the frequency varies linearly with time) can be used for this purpose. This signal is expressed by:

$$
x(t)=A \times \cos \left(2 \pi\left(f_{i}+\beta t\right) t\right) \quad \text { avec } \quad \beta=\frac{f_{f}-f_{i}}{t_{f}}
$$

where $f_{i}$ is the initial frequency at $t=0, f_{f}$ is the final frequency at $t=t_{f}$ and $\beta$ is the rate of frequency increase. The major benefit of using a chirp signal is the possibility of measuring the magnitude ripples in the frequency domain from the modulus of the complex envelope. This is due to the linear relationship between time 
and frequency, if the rate of frequency $\beta$ is small [21]. This would greatly facilitate the implementation and decrease the time of the calibration algorithm.

Fig. 13 shows the block diagram of the STF calibration algorithm. In this first step, each modulator is calibrated separately from the others. Practically, only the output of the modulator which is being calibrated is connected to the digital processing part. The chirp signal, whose frequency varies between the modulator boundaries, is applied to the modulator. This signal can be generated by an analog fractional-N synthesizer [22], but this solution requires some analog components such as a VCO. The proposed optimization algorithm (Fig. 13) uses as input, a one-bit signal generated by a sigma-delta generator such as the one proposed in [23]. The lowpass FIR filter coefficients of Fig. 1) are chosen so that the outside band is rejected sufficiently while its in-band transfer function is flat enough (largest possible bandwidth).

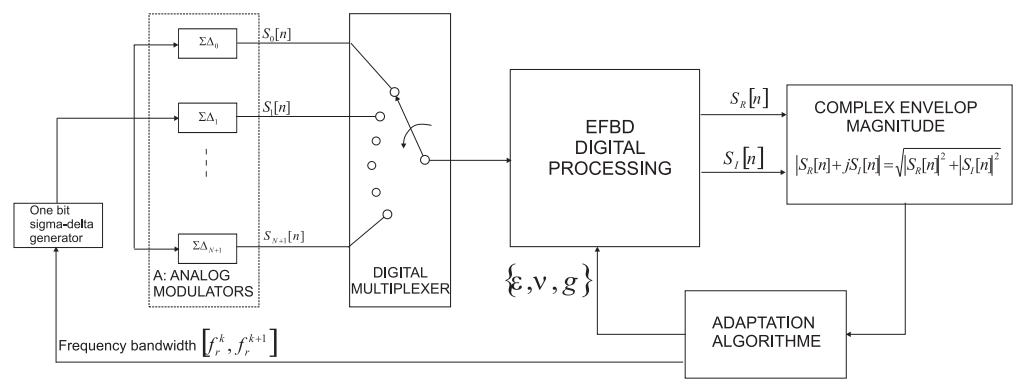

Fig. 13 Block diagram of the STF calibration algorithm.

Fig. 14 summarizes the different steps of the STF calibration algorithm. For each parameter value, a chirp signal is applied covering the useful bandwidths $\left[f_{r}^{k}, f_{r}^{k+1}\right]$; the maximum and minimum output amplitude (the square of the modulus $Q_{k}^{2}+$ $\left.I_{k}^{2}\right)$ are memorized. The difference between the highest amplitude and the lowest amplitude (in-band ripple) is used as the criterion. The optimal parameter $\nu_{\text {opt }}$ is the one that minimizes this ripple.

Fig. 15 shows a simulation example of an EFBD with a single modulator connected to the fourth filter. The input signal is a 1-bit chirp signal varying from reduced frequency $f_{r}^{4}=0.244$ to $f_{r}^{5}=0.256$. The vertical lines show the working band of this modulator (defined by the NTF optimization). The number of the subintervals $N_{v}$ is equal to 16 . A dropout in the STF may be seen before calibration. After calibration, the STF is almost flat.

Even if this algorithm is not optimum in terms of operations, for its practical needs, it only requires counters, registers, a comparator, a sequencer, and an amplitude calculator. With an $800 \mathrm{MHz}$ analog frequency and 40000 samples, each chirp sequence takes $50 \mu \mathrm{s}(40000 / 800 \mathrm{MHz})$ to perform. The execution of the algorithm requires changing the parameters $\nu$ and $\epsilon$ on $N_{v}$ values. With $N_{v}=16$, and as the algorithm converges to the optimal values in 2 iterations, the calibration for each modulator requires $3.2 \mathrm{~ms}(50 \mu \mathrm{s} \times 16 \times 2 \times 2)$ Each execution of the algorithm requires varying the parameter $\nu$ and $\epsilon$ on 16 values. The total STF calibration time for the ten modulators can be estimated at $32 \mathrm{~ms}$. 


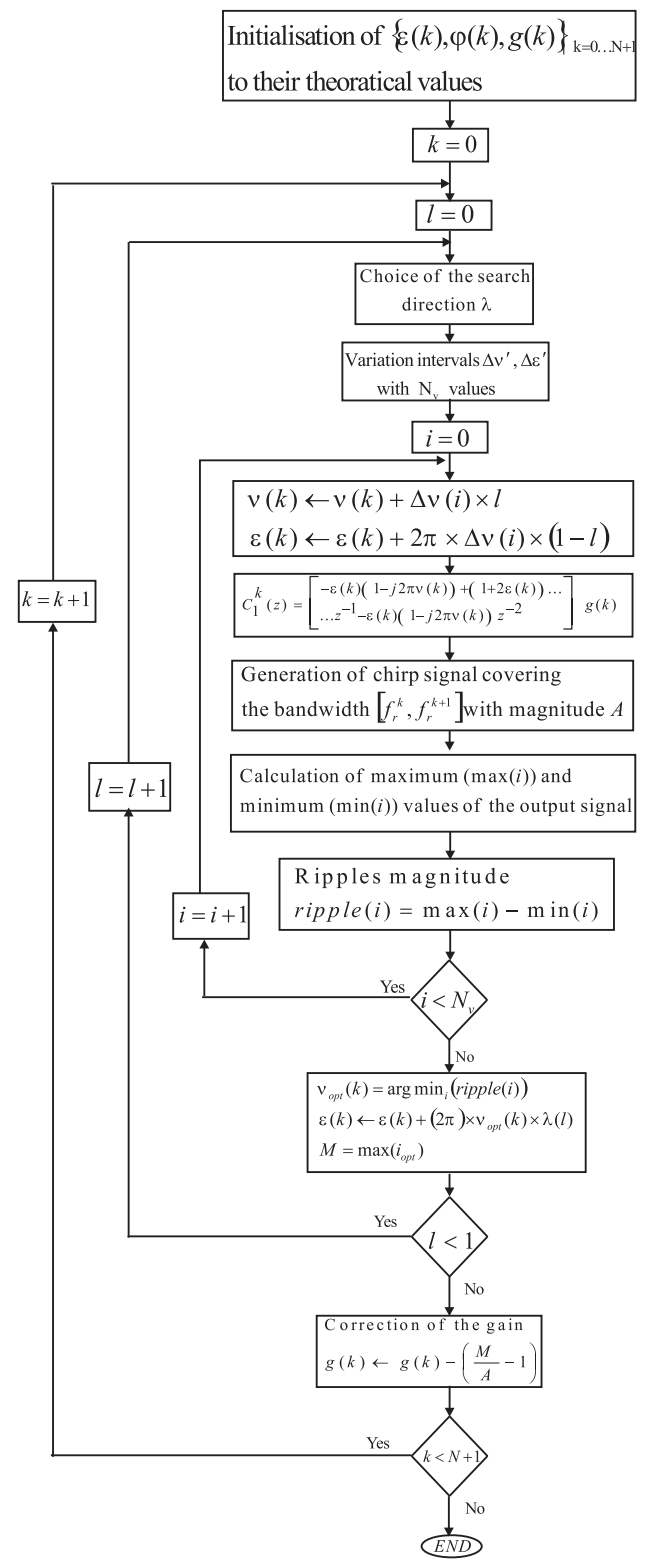

Fig. 14 Flow diagram of the STF calibration algorithm

3.2 Adjusting the phase shifts

Theoretically, the phases are not continuous in the transition zone between adjacent subbands due to the STF phase and to the demodulation and remodulation in the digital part (Fig. 1 - part C). This gap phase introduces ripples around the transition zone in the output spectrum. It has been shown in [5] that this phase discrepancy can be compensated by multiplying the signal in each channel with a constant factor 
a)

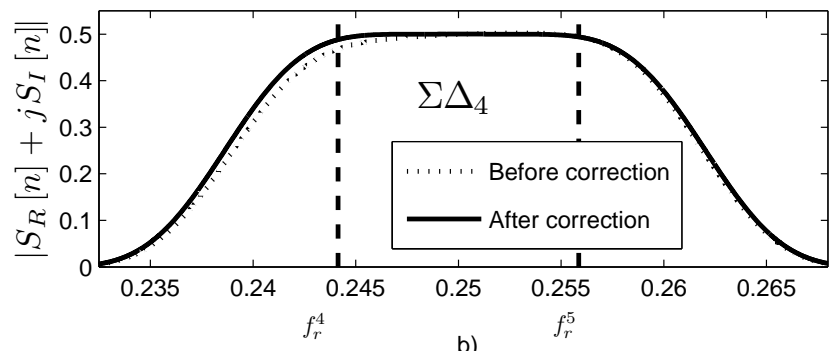

b)

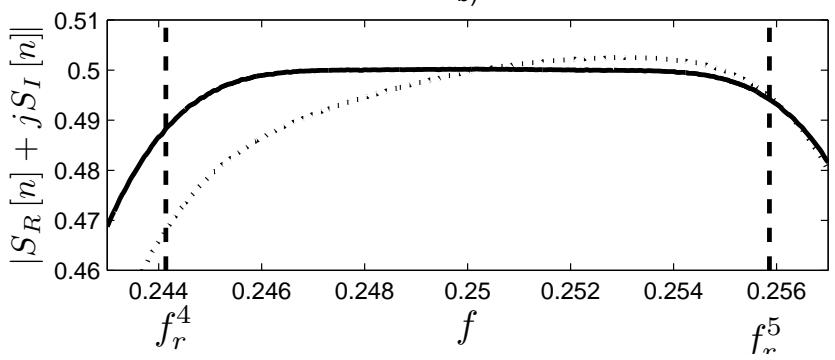

Fig. 15 First phase STF calibration

(a complex number with a modulus equal to unity) in order to obtain a continuous phase in the full band. This multiplication is just a phase shift in the modulation sequence $m_{k}^{\prime}[n]=e^{j 2 \pi N_{d} f_{c}^{k} n}$ (Fig. 1 - part C).

The optimal value of the phase shift is related to the working-band of each channel. It is therefore necessary to recalculate this phase shift following the new bandwidth provided by the NTF optimization algorithm (presented in section 2.2) minimizing the noise power at the output.

The determination of the optimum phase shift value uses the same algorithm for the flattening of the STF. In this case, all the modulators are connected to digital processing. For each channel $k$, the phase shift $d(k)$ varies in an interval $\Delta d$ of $N_{d}$ values around its theoretical value $d_{t h}$ (calculated assuming that the modulators are ideals). Then, for each value of $d$, a chirp signal is generated in the bandwidth $\left[f_{r}^{k-1}, f_{r}^{k+1}\right]$ to measure the ripple magnitudes in the transition zone around the frequency $f_{r}^{k}$ between $\frac{f_{r}^{k}+f_{r}^{k-1}}{2}$ and $\frac{f_{r}^{k}+f_{r}^{k+1}}{2}$. The optimum value is the one minimizing the ripple magnitudes. We choose zero as the phase of the first modulator and we vary the phase shifts of modulators 2 to $N$. Fig. 17 summarizes the different steps of the STF calibration algorithm.

Fig. 16 gives the amplitude of the output of the system for a chirp input signal (amplitude is 0.5 , frequency varies from $0.2 F_{e}$ until $0.3 F_{e}$ ) before and after the calibration. The uncalibrated system has some large ripples $(6.4 \%)$ produced by the phase errors while the response of the calibrated system remains within an acceptable ripple ( $0.4 \%$ below the theoretical value of the module $(0.5))$.

The above algorithm is still a straightforward relaxation algorithm and again, needs only some counters and a sequencer. With an $800 \mathrm{MHz}$ analog frequency and 20000 samples, each chirp sequence takes $25 \mu$ s to perform. If each $d(k)$ value varies 


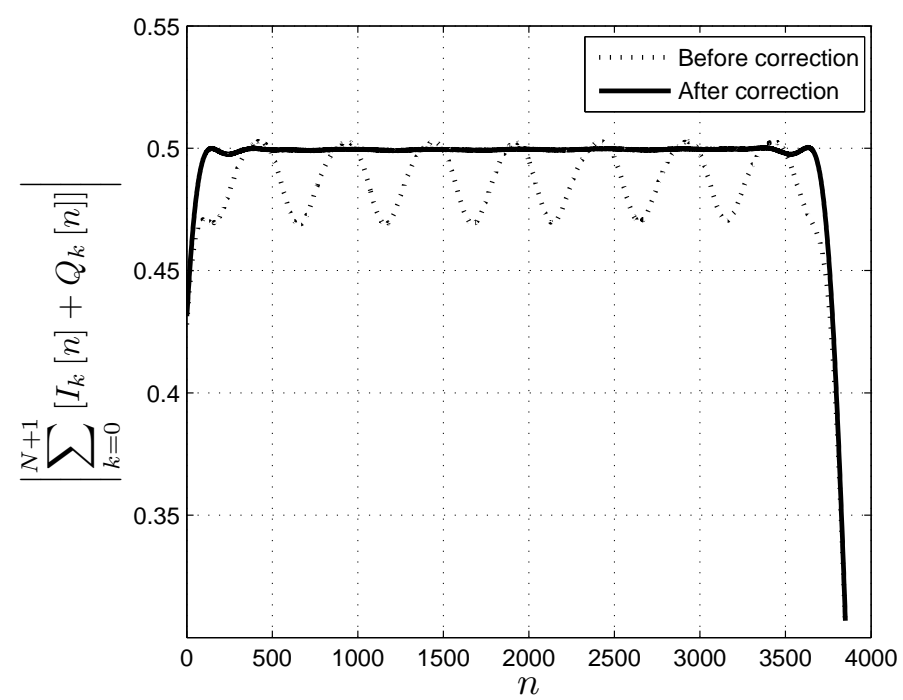

Fig. 16 System output for a chirp input before and after phase calibration

among 64 values, the total phase calibration time for the ten modulators can be estimated to $16 \mathrm{~ms}$.

\section{Conclusion}

Parallelism was used in the most cases in the state of art with time interleaving to increase the ADC bandwidth dedicated to homodyne receiver. The EFBD architecture is the first architecture, to our knowledge, using the frequency band decomposition principle that allows a limited wide band conversion required for heterodyne receivers. We have demonstrated that, with the auto-calibration algorithms described in this paper, the EFBD sigma-delta converter becomes less sensitive to resonator central frequencies shift due to analog imperfections. The main advantage of these algorithms is to use the implemented digital filter plus a reasonable amount of logic. Two main elements are required (a power estimator and a signal generator), associated with counters and a sequencer. The quantity of logic associated with this filter can be neglected when compared with the main filter. The whole calibration process proposed in this paper can be performed in approximately $52 \mathrm{~ms}$, which is compatible with the start-up time of any communications system, even if the proposed algorithm (which is quite simple so as to lead to very low complexity) could be improved in order to decrease this calibration time. Anyway, the possibility of performing a start-up adjustment have shown the feasibility of using a EFBD converter, without requiring rigorous analog adjustments. 


\section{References}

1. P. Aziz, H. Sorensen, and J. Van der Spiegel, "Multiband sigma-delta modulation," Electronics Letters, pp. 760-762, April 1993.

2. P. Aziz, H. Sorensen, and J. Van der Spiegel, "Multiband sigma-delta analog to digital conversion," ICASSP, vol. 3, pp. 249-252, April 1994.

3. A. Eshraghi and T. Fiez, "A comparative analysis of parallel delta-sigma ADC architectures," IEEE Trans. Circuit and Sys.I, vol. 51, pp. 450-458, March 2004.

4. R. Schreier and G.C. Temes, Understanding Delta-Sigma Data Converters. New Jersey: Wiley, 2005, chap. 4

5. P. Benabes, A. Beydoun, and J. Oksman, "Extended frequency-band-decomposition sigmadelta A/D converter," Analog Integrated Circuits and Signal Processing, Springer, 9 January 2009.

6. G. Cauwenberghs, G.C. Temes, "Adaptive digital correction of analog errors in MASH ADCs. I. Off-line and blind on-line calibration," IEEE Transactions on Circuits and Systems II: Analog and Digital Signal Processing, vol. 47, pp 621-628, July 2000.

7. Zhiheng Cao, Shouli Yan, "A digital background calibration method for mash $\Sigma \Delta$ modulators by using coefficient estimation," IEEE International Symposium on Circuits and Systems, Vol. 4, pp 3091-3094, 2005.

8. P. Kiss, Un-Ku Moon, J. Steensgaard, J.T. Stonick, G.C. Temes, "Multibit $\Sigma \Delta$ ADC with mixed-mode DAC error correction," The 2001 IEEE International Symposium on Circuits and Systems, ,vol. 1, pp. 280-283, May 2001.

9. S. Tzi-Hsiung Shu, S. Bang-Sup, K. Bacrania, "A 13-b 10-Msample/s ADC digitally calibrated with oversampling delta-sigma converter," IEEE Journal of Solid-State Circuits, vol. 30, pp. 443-452, April 1995.

10. V. Ferragina, A. Fornasari, U. Gatti, P. Malcovati, F. Maloberti, "Gain and offset mismatch calibration in time-interleaved multipath A/D sigma-delta modulators," IEEE Transactions on Circuits and Systems I: Fundamental Theory and Applications, vol. 51, pp. 2365-2373, Dec. 2004.

11. A. Delic-Ibukic, D.M. Hummels, "Continuous gain calibration of parallel delta sigma A/D converters," IEEE Instrumentation and Measurement Technology Conference, pp. 905-909, April 2006.

12. R.D. Batten, A.Eshraghi, T.S. Fiez, "Calibration of parallel $\Sigma \Delta$ ADCs," IEEE Transactions on Circuits and Systems II: Analog and Digital Signal Processing, ,vol. 49, pp. 390-399, June 2002

13. C. Jabbour, V.T. Nguyen and P. Loumeau, "Optimizing the number of channels for time intrleaved sample-and-hold circuits," in NEWCAS-TAISA 2008, June 2008, pp. 245-248.

14. P. Benabes, M. Keramat, and R. Kielbasa, "A methodology for designing continuous-time sigma-delta modulators," in Proceedings of European design and test conference, March 1997, pp. 46-50.

15. C. Lelandais-Perault, P. Benabes, J.L. De Gouy, and R. Kielbasa, "A parallel structure of a continuous-time filter for band-pass sigma-delta A/D converters," 10th IEEE International Conference on Electronics, Circuits and Systems, pp. 14-17, December 2003.

16. S. Benabid, E. Najafi-Aghdam, P. Benabes, S. Guessab, R. Kielbasa, "CMOS design of a multibit bandpass continuous-time sigma delta modulator running at $1.2 \mathrm{GHz}$, IEEE International Caracas Conference on Devices, Circuits and Systems, pp. 51-55, November 2004.

17. W.R. Bennett, "Spectra of quantized signals," Bell syst. tech. J., vol. 27, pp. 446-472, 1948 .

18. A. Beydoun, P. Benabes, J. Oksman, "Optimization of the Noise Transfer Function of Extended-Frequency Band-Decomposition sigma-delta A/D converters," IEEE Northeast Workshop on Circuits and Systems (NEWCAS'08), pp. 193-196, Montreal, Canada, June 22-25, 2008

19. W.H. Press, B.P. Flannery, S.A.Teukolsky, W.T.Vetterling, NUMERICAL RECIPES, The art of scientific computing. Cambridge University Press 1986

20. M. Ortmanns and F. Gerfers, Continuous-Time Sigma-Delta A/D Conversion. Berlin: Springer, 2006.

21. E. Chassande-Mottin ,P. Flandrin, "On the stationary phase approximation of chirp spectra," $n$ : Proc. of the IEEE Int. Symp. on Time-Frequency and Time-Scale Analysis, pp. 117-120. - Pittsburgh (US), 1998.

22. G. Appel, "Fractional N synthesizers," R.F. Design, vol. 23, pp. 34-50, Nov. 2000. 
23. P.V. Brennan,R. Walkington, "Stored-sequence sigma-delta fractional-N synthesizer," IEE Circuits, Devices and Systems, vol. 151, n 2, pp. 69-73, April 2004. 


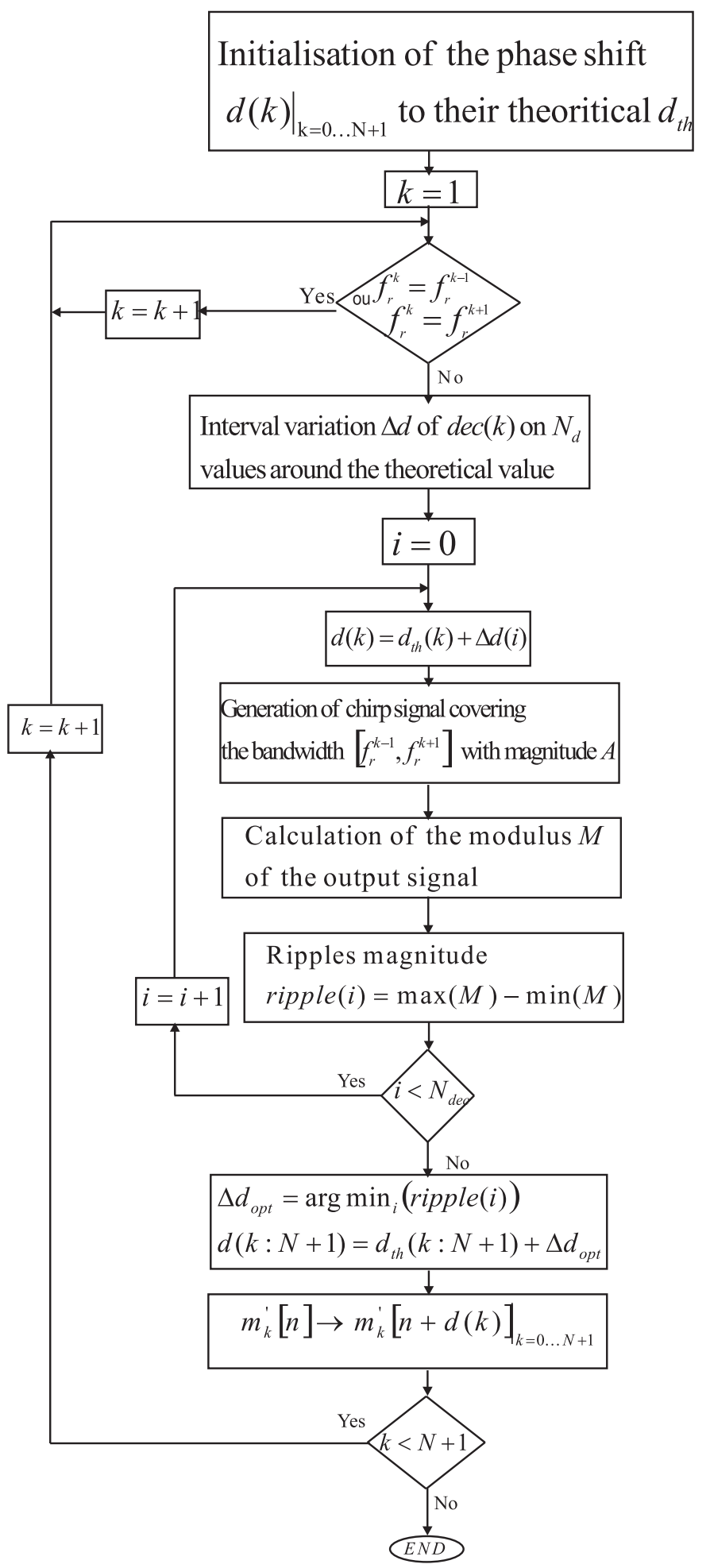

Fig. 17 Flow diagram of the algorithm for phase shift calibration. 